\title{
FATORES QUE PODEM INFLUENCIAR A VIDA DE JOVENS SOROPOSITIVOS
}

\author{
FACTORS THAT MAY INFLUENCE LIFE OF YOUNG \\ SEROPOSITIVES
}

\author{
Pedro Davi Carlos de Moura ${ }^{1}$ \\ Taís Leandra Ferreira dos Santos ${ }^{2}$ \\ Shakira Leticia Fernandes Feitoza ${ }^{3}$ \\ Rafael Tavares Silveira Silva ${ }^{4}$
}

RESUMO: A saúde sexual vai muito além da ausência de Infecções Sexualmente Transmissíveis (IST), embora tal tema tenha se fortalecido como problema de Saúde Pública, pois tem atingido principalmente jovens. Através desse, pretende-se identificar fatores que podem influenciar a vida de jovens que vivenciam o HIVIAIDS. Para tal, lançou-se mão da Revisão Integrativa, a partir da questão norteadora: "Quais fatores tem afetado a vida de jovens soro positivos para o HIV?". Então, durante novembro de 2019 utilizou-se os descritores "Doenças Sexualmente Transmissíveis", "Adolescentes" e "Qualidade de Vida" nas Bases de Dados LILACS, MEDLINE e BDENF, ambas reunidas na Biblioteca Virtual em Saúde. Seguindo critérios de inclusão e exclusão se perfez uma amostra final de 16 artigos, os quais foram analisados criticamente. Percebeu-se, portanto que diversos fatores podem influenciar a vida do desse grupo populacional: o sentimento de vergonha; a fragilidade do serviço de saúde para como este público, expressa na ausência de recursos financeiros e carência de informação e conhecimento específicos sobre HIV/IADS; alcoolismo; tabagismo; drogas; discriminação e exclusão familiar, social e cultural; medo; e problemas psicológicos; agressões físicas, desenvolvendo a necessidade de cuidados físicos e psicológicos; baixa escolaridade; sentimentos quanto ao futuro de incerteza, tristeza, raiva; alteração do padrão de sono. Assim sendo, evidencia-se que o público constituído de jovens e adolescentes soropositivos sofrem influências negativas quanto aos aspectos socioeconômicos,

\footnotetext{
${ }^{1}$ Discentes do curso de Enfermagem da Faculdade Evolução Alto Oeste Potiguar; Pau dos Ferros, Rio Grande do Norte;

${ }^{2}$ Discentes do curso de Enfermagem da Faculdade Evolução Alto Oeste Potiguar; Pau dos Ferros, Rio Grande do Norte;

${ }^{3}$ Discente do curso de Psicologia da Faculdade Evolução Alto Oeste Potiguar; Pau dos Ferros, Rio Grande do Norte;

${ }^{4}$ Enfermeiro. Mestre. Docente do curso de Enfermagem da Faculdade Evolução Alto Oeste Potiguar; Pau dos Ferros, Rio Grande do Norte.
} 
sociais e psicossociais de modo que podem interferir no processo saúde-doença e nas relações sociais.

Palavras chave: HIV. Saúde do Adolescente. Síndrome de Imunodeficiência Adquirida.

ABSTRACT: Sexual health goes far beyond the absence of Sexually Transmitted Infections (STIs), although this theme has strengthened as a Public Health problem, as it has mainly affected young people. Through this, we intend to identify factors that can influence the lives of young people who experience HIV / AIDS. To this end, the Integrative Review was used based on the guiding question: "What factors have affected the lives of young HIV-positive sera?". Then, during November 2019 we used the descriptors "Sexually Transmitted Diseases", "Adolescents" and "Quality of Life" in the LILACS, MEDLINE and BDENF Databases, both gathered in the Virtual Health Library. Following inclusion and exclusion criteria A final sample of 16 articles was made, which were critically analyzed. Therefore, it was noticed that several factors can influence the life of this population group: the feeling of shame; the fragility of health services to this public, expressed in the lack of financial resources and lack of specific information and knowledge about HIV / IADS; alcoholism; smoking; drugs; family, social and cultural discrimination and exclusion; fear; and psychological problems; physical aggression, developing the need for physical and psychological care; low education level; feelings about the future of uncertainty, sadness, anger; change in sleep pattern. Thus, it is evident that the public made up of seropositive young people and adolescents suffer negative influences on socioeconomic, social and psychosocial aspects so that they can interfere with the health-disease process and social relations.

Keywords: HIV. Adolescent Health. Acquired Immunodeficiency Syndrome. 


\section{INTRODUÇÃO}

A adolescência é uma fase peculiar caracterizada por várias alterações físicas e psicológicas na vida do sujeito. Não só o corpo muda com a ação dos hormônios, mas também o comportamento se diferencia daquele quando criança. Inclusive, o interesse por sexo torna- se mais presente (BRASIL, 2013).

O Ministério da Saúde considera adolescente os sujeitos que estão compreendidos entre 10 e 19 anos de idade (BRASIL, 2013). Ou seja, considerando a possibilidade de iniciação sexual nesse período etário, possíveis problemas podem surgir como comportamento sexual de risco, caracterizado por: ausência de camisinha, vários parceiros, gravidez não planejada e Infecções Sexualmente Transmissíveis (IST) (MORAES et al., 2019).

As Infecções Sexualmente Transmissíveis (IST) podem ser definidas como infecções que são transmitidas por contato sexual sem a utilização de preservativo com um sujeito já acometido e que são causadas por diferentes tipos de agentes etiológicos, como o Human Immunodeficiency Virus (HIV), vírus que pode causar a Síndrome da Imunodeficiência Adquirida (AIDS) (BRASIL, 2015).

A AIDS foi descoberta a partir de casos de pessoas com neoplasias e infecções pouco comum e que só eram encontradas em caso relacionados à imunodeficiência (BRASIL, 2015). Então, foi constatado que a existência da síndrome ocorre em decorrência da destruição principalmente dos linfócitos T CD4+ provocada pelo HIV (BRASIL, 2018).

Hoje, embora a Terapia Antirretroviral (TARV) tenha ajudado no controle da doença, já que se observa redução da taxa de detecção, entre a década de 1980 e junho de 2019 já são 966.058 casos registrados, sendo 43.941 novos casos de infecção do vírus e 37.161 da doença só em 2018. Considerando os adolescentes, principalmente homens entre 15 e 19 anos, a taxa de deteç̧ão subiu $62,2 \%$ nos últimos dez anos (BRASIL, 2019). 
Desde o surgimento da pandemia do HIV no Brasil que jovens e adolescentes aparecem nas estatísticas, muito embora identificados como homossexuais, usuários de drogas, profissionais do sexo e nunca como uma faixa etária com comportamentos vulneráveis. Essa invisibilidade implica em distanciamento entre as reais necessidades de saúde sexual e reprodutiva dessa população e as políticas públicas (GRECO, 2016).

Segundo o Ministério da Saúde (BRASIL, 2019), a saúde sexual vai além da ausência de patologias relacionadas ao aparelho reprodutor e ao ato de prazer. São estratégias que promovem a saúde e o desenvolvimento humano, relacionando aspectos sociais, físicos, biológicos e emocionais, sendo capaz de influenciar diretamente a vida das pessoas, em especial o adolescente.

Conhecer, portanto, o contexto em que vive o adolescente soro positivo para o HIV é fundamental para garantir seus direitos sexuais e reprodutivos, além de dignidade. E é nesse ensejo que questiona: quais fatores tem afetado a vida de jovens soro positivos para o HIV?". Assim sendo, pretende-se através desse estudo identificar os fatores que podem influenciar a vida de jovens e adolescentes que vivenciam o HIVIAIDS.

\section{MÉTODO}

O presente estudo lançou mão de uma Revisão Integrativa da Literatura Científica, pois trata-se de um método de síntese de evidências científicas que possibilita a avalição crítica e a interpretação de dados a partir de uma questão norteadora preestabelecida para se chegar ao entendimento de determinado fenômeno (GALVÃO; PEREIRA, 2014).

Constituíram as etapas de elaboração: protocolo de pesquisa; desenvolvimento de uma questão norteadora; localização de estudos a partir da utilização de descritores; seleção e revisão dos estudos levando em consideração critérios de inclusão e exclusão previamente estabelecidos; coleta e síntese dos dados; avaliação crítica dos trabalhos (GALVÃO; PEREIRA; 2014). 
A pesquisa se deu no mês de novembro de 2019, partiu do protocolo que constituiu-se do planejamento prévio constando as demais etapas. A questão que norteou o estudo foi: "Quais fatores tem afetado a vida de jovens soro positivos para o HIV?". E para localização do material usado, foram cruzados através do booleano "AND" os descritores não controlados "Doenças Sexualmente Transmissíveis", "Adolescentes" e "Qualidade de Vida" nas bases de dados eletrônicas LILACS, MEDLINE e BDENF, ambas reunidas no diretório Biblioteca Virtual em Saúde.

A seleção dos artigos seguiu uma lógica sistematizada em fases. Na primeira delas, foram lidos os títulos de todos os artigos encontrados nos cruzamentos “Doenças Sexualmente Transmissíveis X Adolescentes" e "Doenças Sexualmente Transmissíveis X Qualidade deVida”, o que totalizou, respectivamente, 790 e 281 na LILACS, 1.326 e 221 na MEDLINE e por fim 172 e 150 na BDENF. Após a aplicação dos parâmetros de inclusão e exclusão, restaram respectivamente: 28 e 15 artigos na LILACS, 11 e 06 na MEDLINE, 37 e 31 na BDENF.

Os critérios de inclusão foram: publicações nos últimos cinco anos; estudos publicados em português; estudos disponíveis na íntegra e gratuitos; artigos científicos. E os de exclusão: teses, dissertações, artigos de revisão, editoriais, livros; textos incompletos e textos que não respondem a questão de pesquisa.

$\mathrm{Na}$ segunda fase da seleção, os resumos dos 128 artigos foram avaliados com base nos mesmos critérios de inclusão e exclusão, o que possibilitou chegar a seleção 16 da LILACS, dois da MEDLINE e dois da BDENF. E por fim, dos 20 artigos lidos na íntegra, somente 16 compuseram a amostra final, conforme apresenta o Quadro 1.

Para a coleta e síntese dos dados, utilizou-se um formulário contendo informações como nome dos autores, título do periódico, ano de publicação, bases de dado disponível, tipo do estudo e respostas à questão norteadora. O que foi útil para construção de um quadro sinótico no Microsoft Excel for Windows, que posteriormente passou por uma análise crítica à luz da cartilha do Ministério da saúde "Prevenção e Controle das Infecções Sexualmente Transmissíveis, do HIVIAids e das Hepatites Virais" (BRASIL, 2017). 
Quadro 1 - Identificação da amostra final dos artigos selecionados.

\begin{tabular}{|c|c|}
\hline AUTOR/ANO & $\begin{array}{l}\text { BASE DE } \\
\text { DADOS }\end{array}$ \\
\hline $\begin{array}{l}\text { Sistemas bioecológicos e fatores } \\
\text { BRUM et al.,que vulnerabilizam adolescentesTexto \& contexto } \\
2019 \quad \begin{array}{l}\text { frente às infecções sexualmenteenferm. } \\
\text { transmissíveis. }\end{array}\end{array}$ & LILACS \\
\hline $\begin{array}{l}\text { MACIEL et al.,Caracterização do comportamento } \\
2017 \quad \text { sexual entre adolescentes. }\end{array}$ & LILACS \\
\hline 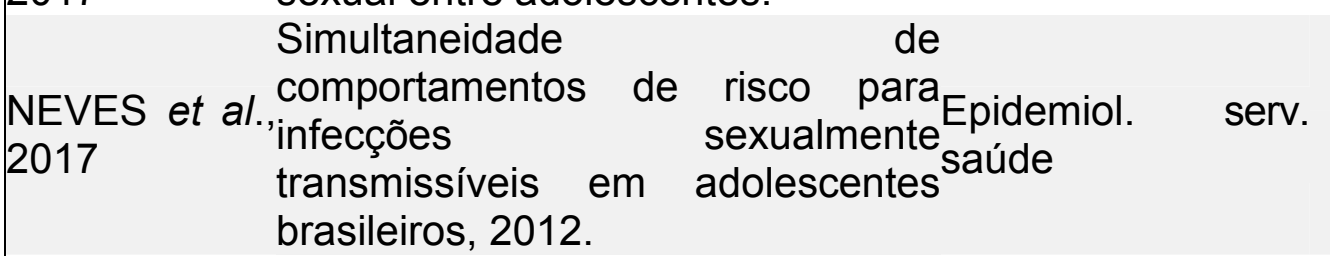 & LILACS \\
\hline \begin{tabular}{l}
\multicolumn{5}{c}{ Conhecimentos, vivências $\mathrm{e}$} \\
SOUZA et al., crenças no campo sexual: um \\
Sostudo com alunos do ensino médio \\
$2017 \quad \begin{array}{lll}\text { REME } \\
\text { com perfis socioeconômicos } \\
\text { diferenciados. }\end{array}$
\end{tabular} & LILACS \\
\hline 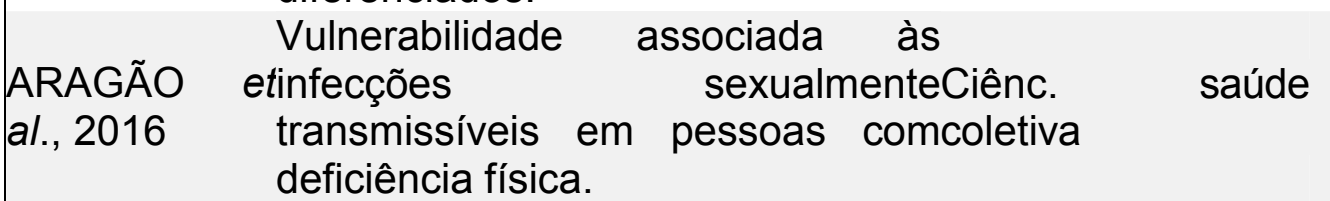 & LILACS \\
\hline $\begin{array}{l}\text { SILVA et al., Ordens e desordens: complexidade } \\
2016 \quad \text { contribuições para enfermagem. }\end{array}$ & LILACS \\
\hline 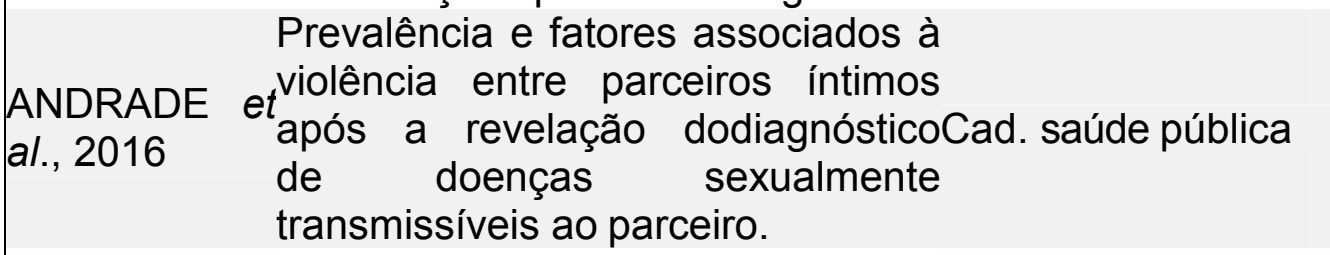 & LILACS \\
\hline \begin{tabular}{ll}
\multicolumn{4}{c}{ Produção de conhecimento acerca } \\
SPINDOLA & etdas doenças sexualmenteRev. pesqui. cuid. \\
al., 2015 & transmissíveis na população jovem:fundam. (Online) \\
& pesquisa bibliométrica.
\end{tabular} & LILACS \\
\hline $\begin{array}{l}\text { RODRIGUES Sexualidade das adolescentes em } \\
\text { et al., } 2015 \quad \begin{array}{l}\text { situação de acolhimento: contextoRev. enferm. UERJ } \\
\text { de vulnerabilidade para DST. }\end{array}\end{array}$ & LILACS \\
\hline $\begin{array}{ll} & \text { Projeto "saúde e prevenção nas } \\
\text { RUSSO; } & \text { escolas": } \text { percepções de } \\
\text { ARREGURY, } & \text { professores e alunos sobre aPhysis (Rio J.) } \\
2015 & \text { distribuição de preservativos } \\
& \text { masculinos no ambiente escolar. }\end{array}$ & LILACS \\
\hline $\begin{array}{lll}\text { CAMPOS et } & \text { a vulnerabilidade ao HIV em } \\
\text { al., } 2014 & \text { edolescentes: estudo retrospectivo } & \\
\text { REME } & \text { rev. } & \text { min. } \\
\text { enferm } & & \end{array}$ & LIL \\
\hline
\end{tabular}


aconselhamento.

GALVÃO et $t_{\text {medicação }}^{\text {Quadidade de vida e adesão à }}$

al., 2015 pessoas com HIV.

FONTES

al., 2017

et Fatores determinantes de

conhecimentos, atitudes e práticasCien Saude Colet. MEDLINE em DST.

Percepções de enfermeiros acerca

SILVA et al.,das vulnerabilidades para DST/AidsRev

2015 diante das conexões do processoEnferm

Gaucha de adolescer.

OLIVEIRA etQualidade de vida de crianças e al., 2015 adolescentes infectados pelo HIV.

Ciênc. cuid. Saúde BDENF

OLIVEIRA Vulnerabilidade de adolescentes às

al., 2018

et doenças transmissíveis na atenção primária.

sexualmente Rev. enferm. UFPE

BDENF

Fonte: Dados produzidos pelos autores da pesquisa.

\section{RESULTADOS}

A partir da análise crítica dos 16 artigos que compõe a amostra final, foi possível perceber que os trabalhos científicos apresentam como o HIV pode influenciar a vida dos jovens soro positivo, através de fatores que podem interferir na sua Qualidade de Vida.

Dentre os fatores pode-se destacar: o sentimento de vergonha; a fragilidade do serviço de saúde para como este público, expressa na ausência de recursos financeiros e carência de informação e conhecimento específicos sobre HIVIAIDS (BRUM et al., 2019; SILVA et al., 2015); alcoolismo; tabagismo; drogas (NEVES et al., 2017; RUSSO, ARREGURY, 2015).

A ausência de informação e hábito de diálogo, impossibilita muitas vezes a utilização do preservativo, tornando o jovem mais vulnerável a IST's (MACIEL et al., 2017; SPINDOLA et al., 2015). Também foi percebido que o receio da família quanto ao diálogo sobre sexo pode favorecer a ausência de informações, o que pode 
aumentar a vulnerabilidade (SOUZA et al., 2017; SPINDOLA et al., 2015; OLIVEIRA et al., 2018).

Pessoas com deficiência físicas, variavelmente possuem poucos parceiros sexuais, isso gerou paradigmas que trazem fatores capazes de influenciar diretamente a vida desses jovens os tornando mais vulneráveis ao HIVIAIDS, tais como: discriminação e exclusão social (ARAGÃO et al., 2016; COSTA et al., 2015).

Jovens que adquiriram o vírus através de uma violência sexual, apresentam fatores como medo, e problemas psicológicos. Pessoas que revelam o diagnóstico do HIV estão mais propensas a sofrer agressões físicas, desenvolvendo a necessidade de cuidados físicos e psicológicos (ANDRADE et al., 2016, SILVA et al., 2016).

O ambiente de vivência define o comportamento individual de cada sujeito, e quando se trata de jovens soropositivos é identificado uma exclusão de ordem familiar, social e cultural, o deixando mais introvertido e inseguro (RODRIGUES et al., 2015, CAMPOS et al., 2014). Os tabus criados em todo das IST tornam-se também um fator agravante. Houve um momento da sociedade que o preservativo era acompanhado de uma série de estigmas, já que havia uma separação entre os que "precisavam" e "não precisavam" usar, tendo em vista que as infecções sexualmente transmissíveis era doenças de pessoas socialmente marginalizadas (RUSSO, ARREGURY, 2015; BRUM et al., 2019).

A vulnerabilidade à Aids é um assunto complexo e que abrange uma grande dimensão, de modo que definem os grupos de maior exposição, o que boa parte das vezes acaba gerando uma maior exclusão e marginalização desses grupos (CAMPOS et al., 2014; RODRIGUES et al., 2015). O fato de a doença ser estigmatizada pela sociedade e de até então não possuir cura pode trazer consequências biopsicossociais ao sujeito acometido, influenciando diretamente sua vida (GALVÃO et al., 2015; OLIVEIRA et al., 2015).

Fatores como alcoolismo e escolaridade baixa são citados como variáveis de alta vulnerabilidade a IST's (FONTES et al., 2017, RUSSO; ARREGURY, 2015). O jovem é um ser vulnerável a partir dos sentimentos de riscos/incertezas que permeiam a adolescência (SILVA et al,. 2015, BRUM et al., 2019). Os jovens soro positivo pode apresentar frequência de medo, tristeza, raiva, alteração do padrão de 
sono e preocupação com o futuro (OLIVEIRA et al., 2015; GALVÃO et al., 2015). Os fatores socioeconômicos podem influencia na qualidade e quantidade de informações sobre a sexualidade do jovem e cuidados com a saúde (OLIVEIRA et al., 2018; SOUZA et al., 2017).

\section{DISCUSSÃO}

A partir dos resultados obtidos foi possível perceber que os artigos corroboram com Reis (2011), quando diz que, a partir do diagnóstico positivo as expectativas do indivíduo em relação a si próprio e em relação ao meio em que vive sofre alterações, repercutindo em âmbito social e pessoal, além de acarretar mudanças no estilo de vida. Segundo Saldanha (2003), após o momento do diagnóstico é necessário que aconteça a reconstrução e manutenção da vida em seus diferentes aspectos.

Gomes (2011), reafirma o que foi apontado nos resultados quando fala que os paradigmas criados em torno da Aids e das pessoas que são portadoras de HIV, exerce cobrança, isolamento e omissão sobre esses sujeitos. Desse modo, contrair o vírus ocasiona uma transgressão social e pessoal e marginalização dos portadores.

Segundo Malbergier e Schoffel (2001), pessoas que são soropositivos podem demonstrar sentimentos de raiva, culpa, autopiedade e ansiedade, o que fundamenta os resultados sobre o jovem estar sempre em situações de riscos/incertezas. De acordo com Brasil (2001), além dos jovens iniciarem a vida sexual cedo, aliam isso a não utilização de preservativos, aumentando a vulnerabilidade a IST's.

De acordo com Cardoso, Malbergier e Figueiredo (2008), os indivíduos alcoolizados têm uma probabilidade maior de praticar sexo sem preservativo, tendo em vista que o índice elevado de álcool interfere na capacidade de discernir os riscos associados a infecções sexualmente transmissível, assim como expostos nos resultados supracitados. Desse modo, compreende-se que fatores adjacentes influencia diretamente a qualidade de vida dos jovens soro positivos. 


\section{CONSIDERAÇÕES FINAIS}

Conclui-se portanto, que aspectos socioeconômicos, sociais, psicossociais e a escolaridade influenciam diretamente a vida dos jovens soro positivos, de modo que interferem no processo saúde-doença e nas relações sociais. Além disso, é necessário que as abordagens da atenção básica de saúde considerem todos os determinantes sociais de saúde no processo de avaliação e cuidado desses sujeitos, de modo que possam oferecer melhores condições de tratamento e cuidado.

Contudo, o presente trabalho conta com um resultado amostral pequeno e com uma discussão limitada a três base de dados. Portanto, é necessário que novos trabalhos continuem a ser desenvolvidos e relatem novas abordagens e perspectivas de jovens soro positivos do vírus e de cuidadores destes sujeitos, de modo que possa facilitar o estudo e trabalho dos profissionais de saúde.

\section{REFERÊNCIAS BIBLIOGRÁFICAS}

ANDRADE, Roumayne Fernandes Vieira et al. Prevalência e fatores associados à violência entre parceiros íntimos após a revelação do diagnóstico de doenças sexualmente transmissíveis ao parceiro. Cadernos de Saúde Pública, Rio de Janeiro, v. 32, n. 7, p.1-10, 2016. FapUNIFESP (SciELO). Disponível em: <http://www.scielo.br/scielo.php?pid=S0102311X2016000705007\&script=sci_abstract\&tlng=pt>. Acesso em: 08 jan. 2020.

ARAGÃO, Jamilly da Silva et al. Vulnerabilidade associada às infecções sexualmente transmissíveis em pessoas com deficiência física. Ciência \& Saúde Coletiva, v. 21, n. 10, p.3143-3152, out. 2016. FapUNIFESP (SciELO). Disponível em: <https://www.scielosp.org/article/csc/2016.v21n10/3143-3152/>. Acesso em: 08 jan. 2020.

BRASIL. Boletim Epidemiológico Especial. Secretaria de Vigilância em Saúde. Ministério da Saúde. AIDS/HIV. Ministério da Saúde, Secretaria de Vigilância em Saúde, Departamento de Doenças de Condições Crônicas e Infecções Sexualmente Transmissíveis - DCCI. Número Especial. Dez. 2019. Brasília: Ministério da Saúde, 2019.

BRASIL. CONITEC. Ministério da Saúde. Secretaria de Vigilância em Saúde. Departamento de DST, Aids e Hepatites Virais. Protocolo Clínico e Diretrizes Terapêuticas: Atenção Integral às Pessoas com Infecções Sexualmente Transmissíveis, - Brasília: Ministério da Saúde, 121p, 2015. 
BRASIL. Ministério da Saúde. Secretaria de Vigilância em Saúde. Recomendações para a Atenção Integral a Adolescentes e Jovens Vivendo com HIV/Aids, - Brasília: Ministério da Saúde, 2013.

BRASIL. Ministério da Saúde. Secretaria de Vigilância em Saúde. Coordenação Nacional de DST e Aids. Boletim Epidemiológico Aids. 27a a 40ª . Semana Epidemiológica. De jun.2001 a set. 2001, - Brasília: Ministério da Saúde, 2001.

BRASIL. Ministério da Saúde. Secretaria de Vigilância em Saúde. Departamento de Vigilância, Prevenção e Controle das Infecções Sexualmente Transmissíveis, do HIVIAids e das Hepatites Virais. Protocolo Clínico e Diretrizes Terapêuticas para Manejo da Infecção pelo HIV em Adultos. Ministério da Saúde, Secretaria de Vigilância em Saúde, Departamento de Vigilância, Prevenção e Controle das Infecções Sexualmente Transmissíveis, do HIVIAids e das Hepatites Virais. Brasília: Ministério da Saúde, 2018.

BRASIL. Ministério da Saúde. Secretaria de Vigilância em Saúde. Departamento de Vigilância, Prevenção e Controle das Infecções Sexualmente Transmissíveis, do HIV/Aids e das Hepatites Virais, - Brasília, Ministério da Saúde, 2017.

BRASIL. Ministério da Saúde. Secretaria de Vigilância em Saúde. Protocolo clínico e diretrizes terapêuticas para atenção integral às pessoas com infecções sexualmente transmissíveis (ist), Brasília: Ministério da Saúde, 2019.

BRASIL. Secretaria Municipal de Saúde. Subsecretaria de Atenção Primária, Vigilância e Promoção da Saúde. Superintendência de Atenção Primária. Infecção pelo HIV e AIDS: prevenção, diagnóstico e tratamento na atenção Primária - v. 1. 1. ed. 83p. Rio de Janeiro: SMS, 2016.

BRUM, Maria Luiza Bevilaqua; MOTTA, Maria da Graça Corso da; ZANATTA, Elisangela Argenta. Bioecological systems and elements that make adolescents vulnerable to sexually transmissible infections. Texto \& Contexto - Enfermagem, v. 28, p.1-13, 1 abr. 2019. FapUNIFESP (SciELO). Disponível em: <https://dx.doi.org/10.1590/1980-265X-TCE-20170492>. Acesso em: 08 jan. 2020.

CAMPOS, Cézar Gustavo Araujo Pacheco de et al. Vulnerability to HIV in adolescents: a retrospective study at a counseling and testing center. Reme: Revista Mineira de Enfermagem, v. 18, n. 2, p.310-314, 2014. GN1 Genesis Network. Disponível em: < http://dx.doi.org/10.5935/1415-2762.20140024.>. Acesso em: 08 jan. 2020.

CARDOSO, Luciana Roberta Donola; MALBERGIER, André; FIGUEIREDO, Tathiana Fernandes Biscuola. O consumo de álcool como fator de risco para a transmissão das DSTs/HIVIAids. Archives Of Clinical Psychiatry (são Paulo), v. 35, p.70-75, 2008. FapUNIFESP (SciELO). Disponível $\quad$ em: $<$ <ttp://www.scielo.br/scielo.php?script=sci_arttext\&pid=S0101-60832008000700015>. Acesso em: 08 jan. 2020.

CRUZ, Elizabete Franco. Crianças, jovens e suas famílias nas esquadrias da epidemia do HIVIAIDS. Desidades, Rio de Janeiro, v. 7, n. 3, 2015, p. 21-32, 2015. Disponível em: < http://desidades.ufrj.br/featured_topic/criancas-jovens-e-suas-familias-nas-esquadrias-daepidemia-do-hivaids/ >. Acesso em: 08 jan. 2020.

FLECK, Marcelo Pio de Almeida. O instrumento de avaliação de qualidade de vida da Organização Mundial da Saúde (WHOQOL-100): características e perspectivas. Ciência \& Saúde Coletiva, [online], v. 5, n. 1, p.33-38, 2000. FapUNIFESP (SciELO). Disponível em: < http://dx.doi.org/10.1590/s1413-81232000000100004>. Acesso em: 08 jan. 2020.

FONTES, Miguel Barbosa et al. Fatores determinantes de conhecimentos, atitudes e práticas em DST/Aids e hepatites virais, entre jovens de 18 a 29 anos, no Brasil. Ciência \& Saúde 
Coletiva, [online], v. 22, n. 4, p.1343-1352, abr. 2017. FapUNIFESP (SciELO). Disponível em: < http://dx.doi.org/10.1590/1413-81232017224.12852015>. Acesso em: 08 jan. 2020.

GALVÃO, Marli Teresinha Gimeniz et al. Qualidade de vida e adesão à medicação antirretroviral em pessoas com HIV. Acta Paulista de Enfermagem, [online], v. 28, n. 1, p.4853, fev. 2015. FapUNIFESP (SciELO). Disponível em: < http://dx.doi.org/10.1590/19820194201500009>. Acesso em: 08 jan. 2020.

GALVÃO, Taís Freire; PEREIRA, Mauricio Gomes. Revisões sistemáticas da literatura: passos para sua elaboração. Epidemiologia e Serviços de Saúde, [online], v. 23, n. 1, p.183-184, mar. 2014. Instituto Evandro Chagas. Disponível em: < http://dx.doi.org/10.5123/s167949742014000100018>. Acesso em: 08 jan. 2020.

GOMES, Antonio Marcos Tosoli; SILVA, Érika Machado Pinto; OLIVEIRA, Denize Cristina de. Social representations of AIDS and their quotidian interfaces for people living with HIV. Revista Latino-americana de Enfermagem, [online], v. 19, n. 3, p.485-492, jun. 2011. FapUNIFESP (SciELO). Disponível em: <http://dx.doi.org/10.1590/s0104- 11692011000300006>. Acesso em: 07 jan. 2020.

GRECO, Dirceu Bartolomeu. Trinta anos de enfrentamento à epidemia da Aids no Brasil, 19852015. Ciência \& Saúde Coletiva, v. 21, n. 5, p.1553-1564, maio 2016. FapUNIFESP (SciELO). Disponível em: <http://dx.doi.org/10.1590/1413-81232015215.04402016 >. Acesso em: 16 out. 2019.

MACIEL, Kellyne Mayara do Nascimento et al. Caracterização do comportamento sexual entre adolescentes [Characteristics of teenage sexual behavior] [Características de la conducta sexual entre adolescentes]. Revista Enfermagem Uerj, [online], v. 25, p.1-7, 25 mar. 2017. Universidade de Estado do Rio de Janeiro. Disponível em: < http://dx.doi.org/10.12957/reuerj.2017.23496>. Acesso em: 07 jan. 2020.

MALBERGIER, André; SCHÖFFEL, Adriana C. Tratamento de depressão em indivíduos infectados pelo HIV. Rev Bras Psiquiatr, [online], v. 3, n. 23, p.160-167, mar. 2001. Disponível em: <http://www.sidastudi.org/resources/inmagic-img/dd1335.pdf>. Acesso em: 07 jan. 2020.

MORAES, Laura de et al. Iniciação sexual precoce e fatores associados: uma revisão da literatura. Psic., Saúde \& Doenças, v. 20, n. 1, p. 59-73. 2019 . Disponível em: http://www.scielo.mec.pt/scielo.php?script=sci_arttext\&pid=S1645_00862019000100005\&ln $\mathrm{g}=$ pt\&nrm=iso. Acesso em: 08 jan. 2020.

NEVES, Rosália Garcia et al. Simultaneidade de comportamentos de risco para infecções sexualmente transmissíveis em adolescentes brasileiros, 2012. Epidemiologia e Serviços de Saúde, [online], v. 26, n. 3, p.443-454, jul. 2017. Instituto Evandro Chagas. Disponível em: < http://dx.doi.org/10.5123/s1679-49742017000300003>. Acesso em: 08 jan. 2020.

OLIVEIRA, Jacqueline Faria et al. Qualidade de vida de crianças e adolescentes infectados pelo hiv / Quality of life of children and adolescents infected with hiv. Ciência, Cuidado e Saúde, [Online], v. 14, n. 1, p.879-884, 27 nov. 2014. Universidade Estadual de Maringa. Disponível em: < http://dx.doi.org/10.4025/cienccuidsaude.v14i1.19265>. Acesso em: 06 jan. 2020.

OLIVEIRA, Patricia Santos de et al. VULNERABILIDADE DE ADOLESCENTES ÀS DOENÇAS SEXUALMENTE TRANSMISSÍVEIS NA ATENÇÃO PRIMÁRIA. Rev Enferm Ufpe On Line, Recife, v. 3, n. 12, p.753-762, mar. 2018. Disponível em: <https://periodicos.ufpe.br/revistas/revistaenfermagem/article/viewFile/25063/28042>. Acesso em: 08 jan. 2020.

REIS, Renata Karina et al. Qualidade de vida, aspectos sociodemográficos e de sexualidade de pessoas vivendo com HIVIAIDS. Texto \& Contexto - Enfermagem, [florionópolis], v. 20, n. 3, 
p.565-575, set. 2011. FapUNIFESP (SciELO). Disponível em: < http://dx.doi.org/10.1590/s010407072011000300019>. Acesso em: 08 jan. 2020.

RODRIGUES, Raquel Fonseca et al. Sexualidade das adolescentes em situação de acolhimento: contexto de vulnerabilidade para DST. Revista Enfermagem Uerj, [rio de Janeiro], v. 23, n. 4, p.507-512, 29 set. 2015. Universidade de Estado do Rio de Janeiro. Disponível em: < http://dx.doi.org/10.12957/reuerj.2015.18402>. Acesso em: 08 jan. 2020.

RUSSO, Kalline; ARREGUY, Marília Etienne. Projeto: percepções de professores e alunos sobre a distribuição de preservativos masculinos no ambiente escolar. Physis: Revista de Saúde Coletiva, [online], v. 25, n. 2, p.501-523, jun. 2015. FapUNIFESP (SciELO). Disponível em: < http://dx.doi.org/10.1590/s0103-73312015000200010>. Acesso em: 05 jan. 2020.

SALDANHA, A. A. W. Vulnerabilidade e Construções de Enfrentamento da Soropositividade ao HIV por Mulheres Infectadas em Relacionamento Estável. Tese de Doutorado. Faculdade de Filosofia, Ciências e Letras de Ribeirão Preto, Universidade de São Paulo, Ribeirão Preto, SP, $2003 . \quad$ Disponível em: $<$ https://teses.usp.br/teses/disponiveis/59/59137/tde-

01102003185727/publico/Tesedoutorado.pdf >. Acesso em: 05 jan. 2020.

SILVA, Ítalo Rodolfo et al. Nurses' perceptions of the vulnerabilities to STD/AIDS in light of the process of adolescence. Revista Gaúcha de Enfermagem, [online], v. 36, n. 3, p.72-78, set. 2015. FapUNIFESP (SciELO). Disponível em: < http://dx.doi.org/10.1590/19831447.2015.03.47293>. Acesso em: 08 jan. 2020.

SILVA, Ítalo Rodolfo et al. Ordens e desordens: complexidade do adolescer e saúde sexual. Revista Enfermagem Uerj, [rio de Janeiro], v. 24, n. 2, p.1-6, 30 abr. 2016. Universidade de Estado do Rio de Janeiro. Disponível em: <http://dx.doi.org/10.12957/reuerj.2016.14569>. Acesso em: 08 jan. 2020.

SOUZA, Vânia de et al. KNOWLEDGE, EXPERIENCES AND BELIEFS IN THE SEXUAL FIELD: A STUDY OF 1ST AND 2ND YEAR HIGH SCHOOL STUDENTS FROM DIFFERENT SOCIOECONOMIC BACKGROUNDS. Reme: Revista Mineira de Enfermagem, [online], v. 21, p.1-11, 2017. GN1 Genesis Network. Disponível em: < http://dx.doi.org/10.5935/14152762.20170001 >. Acesso em: 08 jan. 2020.

SPINDOLA, Thelma et al. The production of knowledge about sexually transmitted diseases in young people: a bibliometric research. Revista de Pesquisa: Cuidado é Fundamental Online, [online], v. 7, n. 3, p.3037-349, 1 jul. 2015. Universidade Federal do Estado do Rio de Janeiro UNIRIO. 3049 Disponível em: < http://dx.doi.org/10.9789/2175- 5361.2015.v7i3.3037->. Acesso em: 08 jan. 2020. 